\title{
GLOSARIO TETRALINGÜE DE TÉRMINOS APLICADOS A LA MORFOLOGÍA Y ANATOMÍA DE OCTOCORALLIA (COELENTERATA: ANTHOZOA)
}

\author{
Annette Calvo Shadid \\ Odalisca Breedy Shadid ${ }^{2}$
}

\begin{abstract}
RESUMEN
Se crea un glosario tetralingue (español, inglés, francés y alemán) de la terminología que se aplica a Octocorallia, con el fin de estandarizar su uso y evitar confusiones a la hora de descibir especies. Las definiciones se dan en español, ya que el glosario está dirigido a los científicos y estudiosos que hablan este idioma. El glosario se divide en cuatro secciones: I. Términos generales de Alcyonacea, II. Forma de las colonias y patrones de ramificación, III. Forma y ornamentación de las escleritas, VI. Índice de referencia en inglés. Se define un total de 180 términos.
\end{abstract}

\begin{abstract}
A Spanish glossary with equivalent terms in three languages (English, Greman, and French) of the terminology (morphology, anatomy, taxonomy) applied to the Octocorallia (Coenlenterata: Anthozoa) was created aiming to standardize its use and facilitate communication among scientists with different languages. Definitions are given in Spanish. This glossary is divided into four sections: I, general terms, including morphology and anatomy; II, growth forms and colonial branching patterns; III, sclarite forms and features; and IV, index of English terms. We define 180 terms.
\end{abstract}

\section{Introducción}

Las primeras publicaciones sobre octocorales, como en la mayoría de los animales, fueron hechas en latín. Eran descripciones muy cortas o simplemente se informaba acerca de un nombre. Conforme el conocimiento de la fauna aumentó, así también la necesidad de hacer descripciones más claras y sistemáticas. Los trabajos más antiguos se escribieron en alemán o francés y luego en inglés. Cada autor usaba una terminología propia. Solamente se contaba con pequeños glosarios muy limitados que se ponían al final de algunas publicaciones para explicar términos que se hubieran usado en el trabajo en particular y en la lengua en que se hubiera escrito la publicación. Estos no pretendían establecer una terminología estándar; por ejemplo, Bayer (1956, 1961), Guzmán y Cortés (1985). A pesar de los esfuerzos de muchos científicos meticulosos, en muchos casos ha habido inconsistencias generadas por la falta de un vocabulario especializado y se ha reconocido que, antes de hacer revisiones sistemáticas y descripciones de especies, es necesario ponerse de acuerdo en cuanto a la terminología morfológica y anatómica 
que se use. En respuesta a esta necesidad, un grupo de especialistas en octocorales, durante el Primer Taller Internacional de Octocorales, celebrado en 1981, decidieron escribir el Illustrated trilingual glossary of morphological and anatomical terms applied to Octocorallia (Bayer et al. 1981), el cual constituye un primer esfuerzo en la estandarización del léxico científico aplicado a octocorales.

Durante el Segundo Taller Internacional de Octocorales, en el año 2002, se llegó a la conclusión de que se debía estimular a los especialistas en octocorales a escribir en inglés, ya que es el lenguaje común entre los científicos. Sin embargo, existe gran cantidad de trabajos regionales que deben hacerse en la lengua correspondiente, por lo que se recomendó el desarrollo de glosarios comprensivos en otros idiomas, por ejemplo, en español. De acuerdo con esto, la presente investigación, además de que constituye un aporte en materia de léxico técnico-científico, suministra un marco de referencia para futuras publicaciones sobre octocorales en idioma español.

\section{Metodología}

El glosario está basado en Bayer et al. (1981). La mayoría de los términos y sus equivalentes en alemán y francés se han tomado de esa publicación. Algunos de los términos son nuevos y de muchos se ha modificado el significado, de acuerdo con recientes discusiones entre expertos (Taller Internacional de Octocorales, 2002). Para algunos términos, no se incluye el equivalente en alguna de las otras lenguas, ya que no han sido usados antes o no se ha decidido cuál es el equivalente. A pesar de algunas diferencias, en general, se sigue un formato parecido al de Bayer et al. (1981) con el fin de que ambos glosarios sean congruentes y complementarios.

El presente glosario se divide en 4 secciones:

I. Términos generales de Alcyonacea.

II. Forma de las colonias y patrones de ramificación.

III. Forma y ornamentación de las escleritas.

VI. Índice de referencia en inglés.

En cada sección, los términos se encuentran ordenados en forma alfabética y las definiciones se tratan en forma enciclopédica. Las referencias cruzadas se presentan cuando existen dos términos para el mismo concepto y se define en una sola de esas formas; en este caso, en la entrada que tenga un uso más común. Para expresar la referencia cruzada entre la entrada en cuestión y la palabra de la referencia, se usa la letra MAYUSCULITA. Como información adicional, en el caso de un término obsolescente, se presenta entre paréntesis antes de indicar la marca que señala la referencia cruzada. En algunas entradas no se da el término equivalente en alguno de los idiomas; esto se debe a que no se han usado en el idioma correspondiente o a que el significado no ha sido establecido por expertos en el idioma en cuestión. 
Siglas

$\begin{array}{ll}m & \text { género masculino } \\ f & \text { género femenino } \\ \text { obs. } & \text { término obsoleto } \\ \text { In. } & \text { inglés } \\ a l . & \text { alemán } \\ f r . & \text { francés }\end{array}$

\section{Términos generales de Alcyonacea}

1. antocodio. $m$. Parte distal del pólipo que incluye la boca, los tentáculos y el cuello. In. anthocodia, al. Anthocodie, fr. anthocodie.

2. antostelio. $m$. Parte proximal, rígida, del cuerpo de algunos pólipos, fortalecida frecuentemente por escleritas, y dentro del cual se puede retraer el antocodio. In. anthostele, al. Anthostele, fr. anthostèle.

3. autozooide. $m$. Pólipo con ocho tentáculos y ocho mesenterios bien desarrollados; es el único tipo de pólipo en las especies monomórficas y son los pólipos de mayor tamaño en las especies dimórficas. In. autozooid, al. Autozooid, fr. autozoide.

4. base. $f$. Porción de una colonia que se adhiere al sustrato. In. holdfast; al. Basis; fr. pied.

5. boca. $f$. Parte superior de la faringe, rodeada por ocho tentáculos. In. mouth; al. Mund; fr. bouche.

6. cáliz. $m$. Proyección cilíndrica del antostelio en forma de verruga. In. calyx, al.Kelch, Polypenkelch, fr. calice.

7. canal gastrodérmico. $m$. Conducto ancho que se origina en los delgados solenia y está conectado con ellos; en algunos casos se usa para denominar la parte basal delgada de la cavidad gástrica de los pólipos. In. gastrodermal canal; al. Gastrodermalkanal; fr. canal gastrodermique.

8. canales longitudinales. $m$. Conductos largos que se extienden paralelamente a lo largo del eje. In. longitudinal canals; al. Längskanäle; fr. canaux longitudinaux.

9. canales separadores. m. Cánulas longitudinales que separan la corteza de la médula en Scleraxonia. In. boundary canals, al. Kranz von Längskanälen, fr. canaux de séparation.

10. cavidad gastrovascular. $f$. Espacio interior de un pólipo que se encuentra dividido radialmente por los mesenterios. In. gastric cavity, gastrovascular cavity; al. Gastralraum; fr. cavité gastrique.

11. celenterón. $m$. = CAVIDAD GASTROVASCULAR. In. coelenteron; al. ; fr.

12. célula. $f$. (obs.) = CÁLIZ. In. cell; al. Zell; fr. cellule.

13. células de la mesoglea. $f$. Hileras de estructuras celulares que cumplen diversas funciones y se encuentran en los vasos celulares de la mesoglea. In. cell strings, al. Zellstränge, fr. chapelets de cellules.

14. cenénquime. $m$. Tejido colonial entre los pólipos, compuesto por mesoglea en la que se encuentran embebidos los pólipos, las escleritas, y los canales gastrodermales. Este tejido está penetrado por la red de solenia. In. coenenchyme; al. coenenchym; fr. coenenchyme. 
15. cnida. $m$. Célula urticante característica de los celenterados. In. cnida; al. Nesselzelle; fr. cnida.

16. cnidoblasto. $m$. Célula de la mesoglea que produce los nematocistos. In. cnidoblast; al. Cnidoblast; fr.cnidoblaste.

17. collar. $m$. Anillo de escleritas que rodea el antocodio en la base de los tentáculos. In. collare; al. Kranz transversaler Spindeln; fr. collier.

18. colonia. $f$. Grupo de pólipos genéticamente idénticos que se encuentran interconectados. In. colony; al. Kolonie; fr. colonie.

19. cordón central. $m$. Parte central del eje en Holaxonia, constituido solo por un material córneo o por material córneo permeado por sustancias calcáreas. En algunas familias, el cordón central es hueco y presenta cámaras o espacios transversales. In. central chord, core; al. Zentralstrang, Achsenstrang; fr. cordon central.

20. corona. $f$. = COLLAR. In. crown; al. Krone; fr. couronne.

21. corteza. $f$. Capa de cenénquime que rodea la médula y contiene los pólipos en los Scleraxonia. En los Holaxonia, representa la capa externa de material córneo que rodea el eje. In. cortex; al. Cortex, Rinde; fr. écorce.

22. corteza axial. $f$. Capa que rodea el cordón central, la cual es depositada por un epitelio axial. In. axis cortex, al. Achsenrinde, fr. cortex axial.

23. cuello. $m$. Parte basal suave y de pared delgada del antocodio, con muy pocas escleritas y que permite la introversión del antocodio dentro del antostelio. In. neck zone, introvert; al. Halszone; fr. cou.

24. dientes caliculares. $m$. Lóbulos poco puntiagudos en el margen del cáliz reforzados con escleritas. Normalmente son ocho, pero en algunos gorgonáceos y penantuláceos son solo dos. In. teeth; al. Kelchzähne, Zähne; fr. dents, dents caliculaires.

25. dimorfismo. $m$. Presencia de dos tipos de pólipos: autozooides y sifonozooides. In. dimorphism; al. Dimorphismus; fr. dimorphisme.

26. disco bucal. $m$. (obs.) = BOCA. In. oral disc; al. mündliche Scheibe; fr. disque buccal.

27. disco oral. $m$. (obs.) = PERISTOMIO. In. oral disc; al. Mundsheibe; fr. oral disque.

28. eje. $m$. Estructura interna de soporte de Gorgonacea y Pennantulacea. Puede ser calcáreo, córneo o poseer ambos componentes. In. axis, al. Achse, fr. axe.

29. epitelio axial. $m$. Capa de células derivadas del ectodermo que producen el eje en Holaxonia. In. axis epithelium, al. Achsenepithel, fr. épithélium axial.

30. esclerita. $f$. Elemento esquelético calcáreo poliforme que se encuentra en la mesoglea y también en el eje de Scleraxonia. In. sclerite; al. Sklerit; fr. sclérite.

31. escleritas suplementarias. $f$. Elementos esqueléticos del antocodio que se encuentran debajo de la corona y están dispuestos en doble fila convergente. Son características en algunas especies de Dendronephthya. In. suplementary sclerites; al. zusätzliche Sklerite; fr. sclérites supplémentaires.

32. escleroblasto. $m$. Célula de la mesoglea que produce una esclerita. In scleroblast; al. Skleroblast; fr. scléroblaste.

33. espícula. $f$. (obs.) = ESCLERITA. In. Spicules; al. Spikeln, Spikula; fr. spicule.

34. estolón. $m$. Expansiones redondeadas, aplanadas o membranosas del cenénquime que crecen periféricamente sobre el substrato o debajo de este y producen nuevos pólipos. In. stolon; al. Stolo; fr. stolon. 
35. estomodeo. $m$. (obs.) = FARINGE. In. stomodaeum; al.Schlundrohr; fr. stomodaeum .

36. faringe. $f$. Conexión tubular entre la boca y la cavidad gástrica. In. pharynx; al. Pharinx; fr. pharinx.

37. filamentos mesentéricos. $m$. = FILAMENTOS. In. mesenterial filaments, al. Mesenterialfilamente, fr. filaments mésentériaux.

38. filamentos. $m$. Bordes engrosados y enrollados de los mesenterios; los dos filamentos de los mesenterios opuestos al sifonoglifo son muy largos y muy flagelados; los seis restantes, son cortos y glandulares. In. filaments; al. Filamente; fr. filaments.

39. hilos. $m$. (obs.) = CNIDA

40. internodo. $m$. Segmento calcáreo duro del eje de algunos Gorgonacea (Melithaeidae, Isididae). In. internode; al. Internodium; fr. internoeud.

41. lado asulcal. $m$. Lado dorsal del pólipo, opuesto al sifonoglifo. In. asulcal side; al. asulcale Seite; fr. coté asulcal (circunflejo).

42. lado frontal. $m$. Lado de la colonia en el cual los pólipos crecen más densamente. In front of a colony; al. Vorderseite; fr. devant.

43. lado sulcal. $m$. Lado del pólipo más cercano a donde se encuentra el sifonoglifo. In. sulcal side; al. sulcale Seite; fr. côté sulcal.

44. lado ventral. $m$. (obs. en relación con el pólipo) = LADO SULCAL

45. lóculo. $m$. Área calcificada semicircular o espacio fibroso del eje de Holaxonia, especialmente en Plexauridae. In. loculus; al. Loculus; fr. locule.

46. médula. $f$. Estructura interna de soporte de los Scleraxonia. In. medulla; al. Zentralstrang; fr. zone centrale compartimentée.

47. mesenterios. $m$. Divisiones delgadas, no calcáreas y radiadas, que unen la faringe a la pared del cuerpo, y dividen la cavidad gastrovascular del pólipo. In. mesenteries; al. Mesenterien; fr. mésentères.

48. mesoglea. $f$. Sustancia gelatinosa que separa las dos capas epiteliales y contiene numerosas células como escleroblastos y células de la mesoglea. Está compuesta de fibras de colágeno embebidas en una matriz amorfa de proteínas altamente hidratadas y polímeros de polisacáridos. In. mesogloea, al.Mesogloea, fr. mésoglée.

49. mesozooide. $m$. Pólipo intermedio entre un autozooide y un sifonozooide. In. mesozooid; al. Mesozooid; fr. mésozoüde.

50. monomorfismo. $m$. Presencia de un solo tipo de pólipo, el autozooide. In. monomorphism; al. Monomorphismus; fr. monomorphisme.

51. nematoblasto. $m .=$ CNIDOBLASTO. In. nematoblast; al. Nematoblast; fr. nematoblaste.

52. nematocisto. $m .=$ CNIDA. In. nematocyst; al. Nesselzelle; fr. nèmatocyste.

53. nematocito. $m .=$ CNIDOBLASTO. In. nematocyt, cnidoblast; al. Cnidoblast; fr. nématoyte, cnidoblaste.

54. nodo. $m$. Articulación córnea flexible del eje calcificado en Melithaeidae y en Isididae; en algunos casos designa el punto de origen de una rama en un tronco no articulado de un gorgónido; en otros, designa el punto de unión donde se anastomosan varias ramas de los abanicos reticulados. In. node; al. Nodium; fr. noeud.

55. opérculo. $m$. En Primnoide, las ocho escamas triangulares que cubren los tentáculos cuando están retraídos. In. operculum; al. Deckel; fr. opercule. 
56. pared corporal. $f$. Pared del cuerpo del pólipo que rodea la cavidad gástrica. In. bodywall, al. Körperwand, fr. paroi polipaire.

57. pedúnculo. $m$. En Alcyonacea y Pennantulacea, parte basal de la colonia que se entierra o embebe en el substrato. En Nephtheidae, parte proximal angosta de un pólipo no retráctil. In. stalk; al. Stiel; fr. pédoncule.

58. peristomio. $m$. Área de un pólipo que rodea directamente la boca, formada por las partes basales internas de los tentáculos. In. peristome; al. Mundregion; fr. péristome.

59. pínulas. $f$. Extensiones laterales de un tentáculo. In. pinnules; al. Pinnulae; fr. pinnules.

60. polimorfismo. $m$. Diferentes tipos de pólipos en una misma colonia. In polimorphism; al. Polymorphismus; fr. polymorphisme.

61. polipario. $m$. Parte de la colonia que contiene el antocodio. In. polyparium; al. Polyparium; fr. polypaire.

63. pólipo axial. $m$. Pólipo más alto de un grupo de ellos que produce pólipos secundarios por medio de yemas laterales que salen de la pared del cuerpo. In. axial polyp; al. Axialpolyp; fr. polype axial.

63. pólipo. $m$. Cada uno de los individuos de una colonia de Octocorallia, independientemente de su estructura anatómica. In. polyp; al. Polyp; fr. polype.

64. pólipo contráctil. $m$. Pólipo que puede reducir su tamaño sin introvertirse. Los tentáculos pueden doblarse hacia adentro sobre la boca, pero no hay un cuello que permita al antocodio retraerse completamente dentro del antostelio o del cenénquime en general. In. contractile polyp; al. kontractiler Polyp; fr. polype contractile.

65. pólipo fundador. $m$. Primer pólipo de una colonia formado por la metamorfosis de la larva plánula. Puede convertirse en un pólipo axial. In. founde polyp; al. Gründerpolyp; fr. polype fondateur.

66. pólipo primario. $m .=$ PÓLIPO FUNDADOR. In. primary polyp, al. Primärpolyp; fr. polype primaire.

67. pólipo retráctil. $m$. Pólipo cuyo antocodio puede introvertirse completamente dentro del antostelio o del cenénquime en general. In. retractile polyp; al. retraktiler Polyp; fr. polype rétractile.

68. puntas. $f$. Ocho líneas de escleritas en doble fila convergente en la parte distal del antocodio. Se encuentran sobre el collar, cuando este está presente. In. Points; al. konvergierende Doppelreihen, Kronenspitzen; fr. doubles reangées, chevrons.

69. rama terminal claviforme. $f$. Rama que se ensancha en su parte terminal en forma de clavo. In. clavae branch, al. verdicktes Zweigende, fr. branche claviforme.

70. reticulación. $f$. $=$ vENTANA

71. rizoides. $m$. Filamentos o falsas raíces que se extienden desde la base de una colonia. In. Rhizoids; al. Rhizoiden; fr. rhizö̈des.

72. $\quad$ septa. $m$. (obs.) = MESENTERIOS

73. sifonoglifo. $m$. Surco fuertemente ciliado que se extiende hacia abajo, de un lado de la faringe. In. siphonoglyph; al. Siphonoglyphe, Flimmerrinne, Wimperrinne; fr. siphonogliphe.

74. sifonozooide. $m$. Pólipo con el sifonoglifo muy desarrollado y con tentáculos reducidos o sin ellos; normalmente presenta filamentos mesenteriales muy pequeños. Este tipo de pólipo es más pequeño que los autozooides. In. siphonozooid; al. Siphonozooi; fr. siphonozoïde. 
75. solenio. $m$. Cada uno de los canales pequeños que penetran el cenénquime formando una red e interconectan las cavidades gástricas de los pólipos y los grandes canales gastrodérmicos. In. solenium; al. Solenium; fr. solénium.

76. sulco. $m$. = SIFONOGLIFO

77. Superficie posterior de la colonia. $f$. Lado de la colonia que contiene pocos pólipos o carece de ellos, o lado contrario de donde se dirige la mayoría de los antocodios. Se aplica normalmente a colonias planas. In. back of a colony, al. Rückseite, fr. derrière.

78. tallo. $m$. En Gorgonacea, parte basal de la colonia de la cual pueden brotar las ramas. En Alcyonacea, parte poliposa de la colonia que usualmente produce las ramas. In. stem; al. Hauptstamm, Stamm; fr. tronc.

79. tentáculos pinados. $m$. Tentáculos rodeados de pínulas que son característicos de Octocorallia. In. pinnate tentacles; al. gefiederte Tentakel; fr. tentacules pinnés.

80. trimorfismo. $m$. Presencia de tres tipos de pólipo en una colonia, además del pólipo primario: autuzooides. sifonozooides y mesozooides. Se da en Pennantulacea y en un género de Alcyonacea. In. trimorphism; al. Trimorphismus; fr. trimorphisme.

81. tronco. $m$. $=$ TALLO.

82. vaina axial. $f$. Parte del cenénquime de la colonia que rodea el eje directamente en Gorgonacea. Usualmente está delimitado por los canales longitudinales y caracterizado por escleritas diferentes a las del resto del cenénquime. In. axial sheath, al. Achsenscheide, fr. gaine axiale.

83. vasos celulares. $m$. Espacios vacíos que se encuentran en la mesoglea. En algunos casos, pueden contener células de la mesoglea. In. zellvessels, al. Zellknäle, fr. vaisseaus cellulaires.

84. ventana. $f$. Espacio encerrado por ramas anastomosadas dentro de la malla. In. mesh, al. Masche, fr. maille.

85. verruca. $f$. (obs.) = CÁLIZ. In. verruca; al. Verruca, Warze; fr. verruca, verrue.

86. zooide. $m$. (obs.) = PÓLIPO. In. zooid; al. Zooid; fr. zooid.

87. zooxantelas. $f$. Algas unicelulares que son simbiontes. In. zooxanthellae; al. Zooxanthellen; fr. zooxanthelles.

\section{Forma de las colonias y patrones de ramificación}

88. arborescente. Colonia ramificada en forma de árbol, que posee un tronco (Gorgonacea ) o un tallo (Alcyonacea). In. arborescent; al. Baumförmige; fr. arborescent.

89. arbustiva. Colonia con ramas abundantes que salen directamente de la base y no forman un tronco definido. In. bushy; al. buschig; fr. buissonant, en buisson.

90. candelabro. $m$. Colonia en un plano cuyas ramas secundarias son verticales y casi paralelas entre sí. In. candelabriform; al. kandelaberförmig; fr. candélabriforme.

91. digitada. Colonia que presenta varios lóbulos alargados como dedos. In. digitate; al. fingerig; fr. digité.

92. digitiforme. Colonia sin ramificar en forma de dedo. In. digitiform; al. fingerförmig; fr. digitiforme.

93. estolonada. Colonia que consiste en varios pólipos interconectados por medio de estolones. In. stolonate; al.stolonat; fr. stoloné. 
94. filiforme. Colonia delgada sin ramificar en forma de hilo. In. filiform; al. fadenförmig; fr. filiforme.

95. flabelado. Colonia en forma de abanico. In. flabellate; al. Fäche; fr. flabelle.

96. flageliforme. Colonia robusta sin ramificar en forma de látigo. In. flagelliform; al. peitschenförmig; fr. flagelliforme.

97. foliado. Colonia sin reticular en forma de hoja. In. leaf-like frond; al. blattförmig; fr. feuilleforme.

98. fungiforme. Colonia sin ramificar con una parte distal amplia y un tallo bastante angosto. In. capitate; al. pilzförmig; fr. capité.

99. hisopo. $m$. Colonia con ramificación pinada, de la cual brotan numerosas ramas cortas alrededor de un tronco principal. In. bottlebrush; al. Flaschenbürstenförmig; fr. brosse à boutelle.

100 incrustada. Colonia que posee una capa carnosa gruesa que cubre el substrato. In. encrusting; al. inkrustierend; fr. encroûtant.

101. liriforme. = CANDELABRO. In. Lyriform; al. lyraförmig; fr. lyriforme.

102. lobulada. Colonia que presenta varios lóbulos robustos. In. lobate; al. in Loben; fr. en lobes.

103. malla. $f$. Ramas que se unen con diferentes niveles de anastomosis para formar las colonias reticulares. In. network; al. Masche; fr. maille.

104. multiplanar. Colonia ramificada cuyas ramas crecen en varios planos. In. multiplanar; al. in mehreren Ebenen; fr. multiplan.

105. pinada. Colonia cuya ramificación tiene forma de pluma y se presenta en un solo plano. In. pinnate; al. federförmig; fr. penné.

106. planar. Colonia cuyas ramas crecen principalmente en un solo plano. In. planar; al.in einer Ebene; fr. dans un plan.

107. ramificaciones terminales. En colonias reticulares, ramas que no se anastomosan en los bordes. In. end-branchlets; al. ende-zweig; fr. extrémité-branche.

108. ramificada dicotómicamente. Colonia ramificada cuyo patrón de ramificación presenta sucesivas bifurcaciones. In. dichotomously branching; al. dichotom verzweigt; fr. ramifié de façon dichotome.

109. ramificada lateralmente. Colonia plana o casi plana cuya ramificación es irregular. In. lateral branching; al. seitlich verzweigt; fr. ramifié latéralement.

110. ramificada. Colonia erguida con ramas; estas pueden consistir en un pólipo primario y otros subordinados. In. branched; al. verzweigt; al. ramifié.

111. ramitas libres. En colonias reticulares, proyecciones sin anastomosar dentro de las ventanas más amplias de la malla. In. free-twigs; al. freistehende Äste; fr.branche detachée.

112. reticular. Colonia cuyas ramas se anastomosan en una estructura en forma de red. In. reticulate; al. netzförmig; fr. rétiforme.

113. sin ramificar. Colonia vertical sin partes laterales. In. unbranched; al. unverzweigt; fr. non-ramifié. 


\section{Forma y ornamentación de las escleritas}

114. aguja. $f$. Eje monoaxial largo, delgado, liso y puntiagudo. In. needle; al. Nadel; fr. aiguille.

115. antorcha. $f$. Mazo con estructuras alargadas en la cabeza y dobladas hacia un lado en forma de antorcha encendida. In. torch; al. Fackel; fr. torche.

116. aristas. $f$. Eje curvo con dos radios largos, puntiagudos y dos protuberancias mediales cortas. In. bracket; al.Klammer; fr. arête.

117. barra. $f$. Esclerita monoaxial recta o curva, truncada en los dos extremos. In. rod; al. Stab; fr. bâtonnet, baguette.

118. barril. $m .=$ DOBLE ESFERA

119. bastón. $m$. Huso bifurcado en un extremo. In. crutch; al. krückenförmiges Sklerit; fr. sclérite en béquille.

120. cabeza doble. $f$. (obs.) = MANCUERNA

121. cabrestante. $m$. Eje radiado con dos espirales de tubérculos o verrugas, con un espacio medial (cintura) definido y dos penachos terminales de protuberancias o verrugas que no llegan a formar una espiral. Se considera cabrestante un eje de ocho o más radios. In. capstan; al. Gürtelstab; fr. cabestan.

122. cabrestante de doble disco. $m$. Tipo de cabrestante con las dos espirales de tubérculos fusionadas en forma de placas. In. double disk, double wheel, birotulate; al. Doppelscheibe, Doppelrad; fr. double disque.

123. canoa. $f$. $=$ ESCAFOIDES

124. capullo. $m$ Esclerita lisa, ancha, fusiforme, con pocas ornamentaciones en forma de pequeñas crestas longitudinales. In. shuttle; al. Webschiffchen; fr. navette.

125. cilindro. $m$. Eje ancho en forma de rodillo, con los extremos truncados y ornamentado con verrugas. In. cylinder; al. Walze; fr. cylindre.

126. cono doble. $m$. (obs.) = HUSO PUNTIAGUDO

127. corona de espinas. $f$. Huso afilado con un extremo puntiagudo liso y el otro espinoso. En conjunto forman una corona que rodea el pólipo en algunas especies de Acanthogorgia. In. crown spines; al. Kronstachel; fr. épine longue circumorale.

128. cruz. $f$. Cuatro radios que se proyectan en un solo plano con las aristas foliadas, espinosas o tuberculadas. In. cross; al. Kreuz, Zwilling, Doppelbildung; fr. croix.

129. cuerno. $m$. Escama pequeña con ramificaciones puntiagudas. In. antler; al. geweihförmige; fr. andouiller, bois.

130. dedito. $m$. Barra pequeña, aplanada y con los bordes lisos. In. finger-biscuitlike form; al. Biskuitförmige Platten.

131. doble esfera. $f$. Esferoide ancho con dos cabezas espinosas o verrucadas y una cintura o espacio medial muy corto. In. Barril, double sphere; al. Fäâchen; fr. tonnelet.

132. escafoides. $m$. Huso curveado en forma de " $C$ ", que puede tener verrugas modificadas en el lado convexo. In. scaphoid; al. Klammer; fr. crampon, scaphö̈de.

133. escama. $f$. Esclerita delgada y plana. In. scale; al. Schuppe; fr. écaille.

134. escama estrellada. $f$. Esclerita plana con los márgenes profundamente lobulados, con simetría radial más o menos definida. In. stellate plate; al. sternförmige Platte; fr. étoile à tubercule, étoilée à bosse centrale. 
135. escama puntiaguda. $f$. Esclerita plana con un proceso central, marginal como espinoso, o digitiforme, pasando por muchas formas transicionales hasta el tipo de escama puntiaguda. In. thornscale; al. Stachelplatte; fr. plaque à aiguillon.

136. escamas basales. $f$. Par de escleritas corporales más proximales en los géneros Narella y Paracalyptrophora (Primnoidae). In. basal scales; al. Basalschuppen; fr. sclérites de la base.

137. escamas bucales. $f$. Par de escleritas corporales más distales que se encuentran encima de las escamas operculares en los géneros Narella y Paracalyptrophora (Primnoidae). In. buccal scales; al. Bukkalschuppen; fr. sclérites buccaux.

138. escamas infrabasales. $f$. Uno o más pares de pequeñas escamas corporales que se encuentran entre las escamas basales y las escleritas del cenénquime en los géneros Arthrogorgia, Calyptrophora y Paracalyptrophora (Primnoidae y Chrysogorgiidae). In. infrabasal scales; al. Infrabasalschuppen.

139. escamas mediales. $f$. Par de escleritas (en algunos casos dos pares) que se encuentra entre las escamas basales y las bucales en los géneros Narella y Paracalyptrophora (Primnoidae). In. medial scales; al. Medialschuppen; fr. sclérites du milieu.

140. escamas operculares. $f$. Escleritas corporales más proximales que se encuentran debajo de las escamas bucales en los géneros Narella y Paracalyptrophora (Primnoidae). In. opercular scales; al. Rumpfschuppen.

141. esfera. $f$. Esclerita redondeada parcial o completamente. Por lo general se combina con otro término para definir sus características, como esferoide foliado o esferoide espinoso. In. ball, spheroid; al. Kugel; fr. boule.

142. esfera foliada. $f$. Estructura esférica con un lado modificado en forma de hojas. In. foliate spheroid; al. Blattkugel; fr. sphère foliacée, globule foliacée.

143. esfera tuberculada. $f$.= ESFERA FOLIADA

144. espícula. f. Esclerita monoaxial tipo aguja. In. spicule; al. Spikeln, Spicula; fr. spicule.

145. estrella doble. $f$. Eje simétrico con dos extremos de proyecciones tuberculadas o espinosas y con un espacio medial liso bien definido. In. double star; al. Doppelstern; fr. double étoile.

146. estrella puntiaguda. $f$. Esclerita que resulta de la modificación del tipo escama puntiaguda; en ella, algunos de los procesos espinosos se proyectan verticalmente por la parte central dividida de la esclerita y forman prolongaciones tipo raíz en la base y la parte superior es más bien ancha en forma de hoja. In. thronstar; al. Stachelfuß; fr. étoile à aiguillon.

147. heptarradiada. $f$. Eje con dos anillos de tres tubérculos cada uno y uno más en un solo extremo. In. seven-radiate; al. Sievenstrahler; fr. heptaradiaire.

148. hexarradiada. $f$. Eje central con dos anillos terminales de tres tubérculos cada uno. In. six-radiate; al. Sechsstrahler; fr. hexaradiaire.

149. hoja con raíz. $f$. Esclerita en forma de mazo. Consiste en una cabeza discoidal o lubulada y una raíz tuberculada. In. rooted leaf; al. Wurselblatt; fr. feuille à racines.

150. huso. $m$. Esclerita monoaxial recta o curveada, por lo general tiene un espacio medial angosto como una cintura, con ornamentación muy variable, muy común. In. spindle; al. Spindel; fr. fuseau. 
151. huso con espuela. $m$. Eje con un proceso tipo espina a un lado. In. thornspindle; al. Stachelspindel; fr. fuseau à aiguillon.

152. huso de discos. $m$. Eje con cuatro o más espirales de tubérculos fusionados casi por completo en forma de láminas planas irregulares. In. disk-spindle; al. Scheibenträger; fr. fuseau à disques.

153. huso doble. (obs.) = HUSO PUNTIAGUDO

154. huso espinoso. $m$. Eje normalmente curveado con múltiples proyecciones espinosas unilateralmente, algunas de ellas alargadas en forma de ramas. In. branched spindle; al. verzweigte Spindel; fr. fuseau ramifié.

155. huso foliado. $m$. = HUSO ESPINOSO

156. huso puntiagudo. $m$. Eje con los extremos agudos o puntiagudos. In. spindle with acute ends; al. Doppelspindel; fr. double fuseau.

157. huso romo. $m$. Eje con los extremos truncados o redondeados. In. spindle with blunt ends; al. Doppelspindel; fr. double fuseau.

158. lazo. $m$. Estructura plana y acinturada con los extremos distales tuberculados. In. bow; al. Schleife; fr. ruban.

159. mancuerna. $f$. Eje simétrico con dos cabezas más o menos esféricas, verrucosas o tuberculadas con un espacio medial o cintura lisa, más larga y marcada que en la doble esfera. In. dumb-bell; al. Doppelkugel; fr. boule double, haltére.

160. mariposa. $f$. Eje con cuatro proyecciones radiadas, ornamentadas con espirales tuberculadas o verrugas, de las cuales dos son más cortas. In. butterfly-form; al. Schmetterlingsform; fr. forme en papillon.

161. mazo. $m$. Eje con un extremo ancho que puede tener varias formas (la cabeza) y con el otro extremo delgado (la agarradera). In. club; al. Keulen; fr. massues.

162. mazo espinoso. $m$. Eje con la cabeza ornamentada con proyecciones puntiagudas, filosas, o espinosas. In. thorn club; al. Stachelkeule; fr. massue à épines.

163. mazo foliado. $m$. = ANTORCHA

164. mazos gemelos. $m$. Doble esfera con radios enormemente ensanchados (dos cabezas) en un extremo y otros dos cortos y tuberculados en el otro (las agarraderas). In. double club; al. Doppelkeule; fr. double massue.

165. mazo pelón. $m$. Eje corto con pocas proyecciones lobuladas lisas en la parte distal o cabeza y con algunas espinas en la base o agarradera. In. leptoclados-type club.

166. mazo redondo. $m$. Eje ancho con un extremo casi liso, esferoidal o lobulado (la cabeza) y con una base larga, ornamentada con protuberancias bajas o con dos o tres espirales de espínulas alrededor de la parte media del otro extremo (la agarradera). In. balloon club; al. Ballonkeulle, dütenförmige Spicula; fr. massue arrondie.

167. mazo verrucoso. $m$. Eje con la cabeza ornamentada con verrugas o con tubérculos verrugosos, con la agarradera aguda y también verrugosa. In. wartclub; al. Warsenkeule; fr. massue verruqueuse.

168. media luna. $f$. Huso arqueado en forma de "C", con protuberancias más o menos uniformes, sin modificaciones o reducciones en el lado convexo. In. crescent; al. halbmondförmiges Sklerit; fr. croissant. 
169. medusa. $f$. Esclerita tipo mazo formada por una cabeza gruesa y redondeada y una base con proyecciones en forma de raíz. In. rooted head; al. Wurselkopf; fr. tête à racines.

170. octorradiada. $f$. Cabrestante con dos espirales de tres tubérculos cada una y proyecciones terminales. In. eight-radiate; al. Achtstrahler; fr.cabestan octoradié.

171. oruga. $f$. Huso ligeramente curvo con espinas largas de un solo lado. In. caterpiller, unilaterally spinose spindle; al. Raupe; fr. chenille.

172. ovalada. $f$. Eje corto muy ornamentado con bordes suavemente redondeados. In. oval, ovoid; al. Ellipsoid, Oval, eirund; fr. ovale.

173. palo de hockey. $m$. Huso que forma un ángulo obtuso con una parte distal alargada y una parte proximal corta. In. hockey-stick spindle; al. Hockeyschläger; fr. crosse.

174. placa. $f$. Estructura plana, más gruesa que la escama, con diversas formas que van desde circulares y ovaladas hasta poligonales y con bordes comúnmente irregulares. Usualmente mide más de $0.05 \mathrm{~mm}$. In. plate; al. Platte; fr. plaque.

175. plaquita. $f$. Estructura plana pequeña que presenta diferentes formas y es aparentemente lisa, pero con una fina ornamentación granulosa. Usualmente mide menos de $0.05 \mathrm{~mm}$. In. platelets; al. Plättchen; fr. plaquettes.

176. radiada. $f$. Eje principal que se proyecta simétricamente en diferentes planos. In. radiate.

177. roseta. $f$. Eje asimétrico como una copa con un extremo ancho formado por pequeñas espínulas y con una base pequeña redonda y multituberculada. In. double cup, rosette, spiny rosette; al. kelchförmiges Sklerit; fr. rosette, double rosette, corbeille, rosette à épines.

178. tipo Villogorgia. $f$. Ejes aplanados, algo torcidos, con forma de boomerang, con el borde convexo aserrado cerca del lado más ancho, se encuentra en la parte proximal de los tentáculos. In. Villogorgia-type sclerite; al. Drachenflügel; fr. Villogorgia-type.

179. trirradiada. $f$. Eje con tres proyecciones radiales más o menos en el mismo plano. In. triradiate; al. Dreistrahler; fr. triradiaire, étoile à trois pointes, trépied.

180. tubérculos. $m$. Proyecciones simétricas o asimétricas de las escleritas que pueden ser lisas o verrugosas y se encuentran dispersas o acomodadas en forma de anillos. In. tubercle.

\section{VI. Índice de referencia en inglés}

$\begin{array}{lrlr}\text { anteler } & 129 & \text { axis sheath } & 29 \\ \text { anthocodie } & 1 & \text { axis } & 28 \\ \text { anthostele } & 2 & \text { back of a colony } & 77 \\ \text { arborescent } & 88 & \text { ball } & 141 \\ \text { asulcal side } & 41 & \text { ballon club } & 166 \\ \text { autozooid } & 3 & \text { bark } & 22 \\ \text { axial polyp } & 63 & \text { barrel } & 118,131 \\ \text { axial sheath } & 82 & \text { basal scales } & 136 \\ \text { axis cortex } & 22 & \text { birotulate } & 121 \\ \text { axis epitelium } & 29 & \text { body wall } & 56\end{array}$




\begin{tabular}{|c|c|c|c|}
\hline bottlebrush & 99 & double club & 164 \\
\hline boundary canals & 9 & double cone & 126 \\
\hline bow & 158 & double cup & 177 \\
\hline bracket & 116 & double disk & 122 \\
\hline branched spindle & 154 & double head & 120 \\
\hline branched & 110 & double sphere & 131 \\
\hline buccal scales & 137 & double spindle & 152 \\
\hline bushy & 89 & double star & 145 \\
\hline butterfly-form & 160 & double wheel & 121 \\
\hline calyx & 6 & dumb-bell & 159 \\
\hline candelabriform & 90 & eight-radiate & 170 \\
\hline canoe & 123 & encrusting & 100 \\
\hline capitate & 98 & end-branchlets & 107 \\
\hline capstan & 121 & filaments & 38 \\
\hline caterpillar & 171 & filiform & 94 \\
\hline cell strings & 13 & finger-biscuitlike form & 130 \\
\hline cell vessels & 83 & flabellate & 95 \\
\hline cell & 12 & flageliform & 96 \\
\hline central chord & 19 & foliate spheroid & 142,143 \\
\hline clavate branch & 69 & founder polyp & 64 \\
\hline club & 161 & free-twigs & 111 \\
\hline cnematocyst & 15,52 & front of a colony & 42 \\
\hline cnida & 15,39 & gastric cavity & 10 \\
\hline cnidoblast & 16 & gastrodermal canal & 7 \\
\hline coelenteron & 11 & hockey-stick spindle & 173 \\
\hline coenenchyme & 14 & holdfast & 4 \\
\hline collaret & 17 & infrabasal scales & 138 \\
\hline colony & 18 & internode & 40 \\
\hline contractil polyp & 64 & lateral branching & 109 \\
\hline core & 19 & leaf club & 162 \\
\hline cortex & 21 & leaf spindle & 155 \\
\hline crescent & 168 & leaf-like frond & 97 \\
\hline cross & 128 & leptoclados-type club & 165 \\
\hline crown and points & 20,68 & lobate & 102 \\
\hline crown spines & 127 & loculus & 45 \\
\hline crown & 20 & longitudinal canals & 8 \\
\hline crutch & 119 & lyriform & 101 \\
\hline cylinder & 125 & medial scales & 139 \\
\hline dichotomously & & medulla & 46 \\
\hline branching & 108 & mesenterial filaments & 37 \\
\hline digitate & 91 & mesenteries & 47 \\
\hline digitiform & 92 & mesh & 70,84 \\
\hline dimorphism & 25 & mesoglea & 48 \\
\hline disk-spindle & 152 & mesozooid & 49 \\
\hline
\end{tabular}




\begin{tabular}{|c|c|c|c|}
\hline monomorphism & 50 & shuttle & 124 \\
\hline mouth & 5 & siphonoglyph & 73,76 \\
\hline multiplanar & 104 & siphonozooid & 74 \\
\hline neck zone & 23 & six-radiate & 148 \\
\hline needle & 114 & solenium & 75 \\
\hline nematoblast & 51 & spheroid & 143 \\
\hline nematocyte & 52 & spicule & 33,144 \\
\hline network & 103 & spicules & 144 \\
\hline node & 54 & spindle & 150 \\
\hline opercular scales & 140 & spindle with acute ends & 156 \\
\hline operculum & 55 & spindle with blunt ends & 157 \\
\hline oral disc & 26,27 & spiny rosette & 177 \\
\hline oval & 172 & stalk & 57 \\
\hline ovoid & 172 & stellate plate & 134 \\
\hline peristome & 58 & stem & 78,79 \\
\hline pharynx & 36 & stolon & 34 \\
\hline pinnate tentacles & 80 & stolonate & 93 \\
\hline pinnate & 105 & stomodaeum & 35 \\
\hline pinnules & 59 & sulcal side & 43,44 \\
\hline planar & 106 & sulcus & 76 \\
\hline plate & 174 & supplementary sclerites & 31 \\
\hline platelets & 175 & teeth & 24 \\
\hline points & 68 & thorn club & 162 \\
\hline polymorphism & 60 & thornscale & 135 \\
\hline polyp & 62 & thornspindle & 151 \\
\hline polyparium & 61 & thornstar & 146 \\
\hline primary polyp & 66 & thread cell & 39 \\
\hline radiates & 176 & torch & 115,163 \\
\hline reticulate & 112 & trimorphism & 81 \\
\hline retractil polyp & 67 & triradiate & 179 \\
\hline rhizoids & 71 & trunk & 78 \\
\hline rind & 28 & tuberculate & 180 \\
\hline rod & 117 & unbranched & 113 \\
\hline rooted head & 169 & unilaterally spinose & \\
\hline rooted leaf & 149 & spindle & 171 \\
\hline rosette & 177 & verruca & 85 \\
\hline scale & 133 & Villogorgia-type & \\
\hline scaphoid & 123,132 & sclerite & 178 \\
\hline sclerite & 30 & wart club & 167 \\
\hline scleroblast & 32 & zooid & 86 \\
\hline septa & 72 & zooxanthellae & 87 \\
\hline seven-radiate & 147 & & \\
\hline
\end{tabular}




\section{Notas}

1. Escuela de Filología y Lingüística, Universidad de Costa Rica.

2. Centro de Investigación en Ciencias del Mar y Limnología (CIMAR).

\section{Bibliografía}

Bayer, F. M. 1956. "Octocorallia". En Moore, R. C. (ed.).

1961. The shallow water Octocorallia of the West Indian Region. A manual for marine biologists. Martinus Nijhoff, The Hauge, 400 p.

Bayer, F. M., M. Grasshoff y J. Verseveldt. 1983. Illustrated trilingual glossary of morphological terms applied to Octocorallia. E.J. Brill, Leiden. $74 \mathrm{p}$.

Guzmán, H. y J. Cortés. 1985. "Organismos de los arrecifes coralinos de Costa Rica VI. Descripción y distribución geográfica de octocoralarios (Cnidaria) de la costa Caribe". Brenesia, 24: 125-74.

Moore, R. C. (ed.). 1956. Treatise on Invertebrate Paleontology. Part F. Kansas: University of Kansas Press. F166-F231.

Svensén, B. 1993. Practical Lexicography. Oxford: Oxford University Press. 50p. 